\title{
RÔLE DES MUTATIONS DANS L'ACTIVATION DES ONCOGÈNES ET DANS L'INACTIVATION DES GENES SUPPRESSEURS DE TUMEURS
}

\author{
Alain SARASIN \\ Laboratoire de Génétique Moléculaire, \\ Institut de Recherches Scientifiques sur le Cancer \\ BP. $n^{\circ} 8,94801$ Villejuif, France
}

L'acquisition du phénotype tumoral est un processus complexe et long qui recquiert plusieurs étapes cellulaires et la modification de nombreux gènes. il existe au moins deux grandes catégories de gènes impliqués dans la transformation tumorale : les oncogènes dont l'action est d'entraîner les cellules normales vers un phénotype malin et les gènes supresseurs de tumeurs (antioncogènes) dont l'action est de retarder ou d'inhiber la transformation. La plupart des tumeurs humaines ont une ou plusieurs mutations (au sens large du terme) sur l' une ou les deux classes de ces gènes.

Parmi les proto-oncogènes cellulaires dont l'activation en oncogènes se fait par mutation ponctuelle, les gènes de la famille ras sont de loin les plus représentés. En effet, 15-20\% des tumeurs humaines (tous cancers confondus) possèdent au moins une mutation sur l'un des trois gènes ras. Les points chauds de mutagénèse sont limités aux codons 12, 13 et 61. La plupart des mutations ponctuelles sur ces sites induisent un changement localisé de la structure tridimensionnelle de la protéine ras se traduisant en particulier par la perte de l'activité GTPasique dont le rôle est fondamental dans la transmission d'information à travers la membrane cytoplasmique. Parmi les tumeurs cutanées induites par l'irradiation solaire on en dénombre environ $20 \%$ qui possèdent une mutation ponctuelle sur l'un des trois gènes ras. Ce chiffre dépasse les $50 \%$ lorsque les tumeurs ont été isolées de malades atteints de xeroderma pigmentosum, c'est-à-dire déficient en réparation de l'ADN (Suarez et al., $1987 ; 1989)$. 
La nature et le rôle des lésions sur l'ADN dans l'induction des mutations sur les gènes ras peuvent être abordés de façon plus précise en traitant in vitro par un agent mutagène un gène ras cloné. Le gène endommagé est ensuite transfecté dans une cellule cible de souris NIH3T3 et la sélection pour la formation de foyers de cellules transformées par le gène lésé permet de mesurer une efficacité de mutagénèse. L'irradiation UV du gène $N$-ras traité ou non par une enzyme qui enlève spécifiquement certaines lésions permet de démontrer que les dimères de pyrimidines sont directement responsables de la production de mutation (type $T$ vers $A$ ou $C$ vers $T$ ) sur cet oncogène et donc partiellement à la base de la transformation cellulaire (Van der Lubbe, 1988). Ces classes de mutations sont aussi retrouvées sur les oncogènes activés dans les tumeurs de la peau (Daya-Grosjean et al., $1993)$.

L'hypothèse des gènes suppresseurs de tumeurs a été faite suite aux expériences de régression d'un phénotype tumoral après fusion cellulaire entre cellules normales et cellules tumorales. L'étude de certaines maladies génétiques associées de façon mendélienne à un taux de cancers très élevé a permis la mise en évidence et la caractérisation de certains gènes suppresseurs. Ainsi, le rétinoblastome familial est dû à la perte de l'activité du gène $\underline{\mathrm{rb}}$ (souvent lié à une délétion plus ou moins partielle du gène), tandis que le syndrome de Li- Fraumeni est dû à la présence de mutations ponctuelles sur le gène P53. Les produits de ces deux gènes sont tous les deux impliqués dans le contrôle du cycle cellulaire normal en particulier au niveau du passage G1/S (Hartwell, 1992). Leur absence d'activité entraine un dysfonctionnement du cycle cellulaire caractéristique des cellules tumorales. En cas de lésions sur l'ADN, la protéine P53 est stabilisée ce qui entraîne un blocage du cycle cellulaire en G1 et donc un temps supplémentaire pour effectuer la réparation de l'ADN.

Dans le cas de l'ataxie télangiectasia (AT), les cellules traitées aux radiations ionisantes n'ont pas d'augmentation de stabilité de la protéine P53, ce qui fait qu'il n'existe pas de blocage du cycle cellulaire dans ces cellules. Celles-ci rentrent donc en phase $S$ malgré la présence de lésions non réparées et cette étape est sûrement liée à l'hypersensibilité des cellules AT aux radiations ionisantes et peut-être au taux élevé de cancers chez les malades AT (Kastan et al., 1992). 
La perte de l'activité de régulation liée à la protéine P53 sauvage est souvent due à une mutation ponctuelle sur son gène. Environ $50 \%$ des cancers humains possèdent une mutation sur un des allèles du gène P53. Plus de 1200 mutations isolées de tumeurs humaines multiples ont été analysées et environ 100 sites potentiels de mutations sont connus sur ce gène (Caron de Fromentel and Soussi, 1992). Ce très large spectre de sites mutables fait du gène P53 une cible intéressante pour relier les types de mutations observés dans une tumeur donnée et l'éventuel agent cancérogène. Ainsi, dans le cas des cancers hépatiques que l'on trouve en Afrique probablement liés à la présence simultanée du virus de l'hépatite $B$ et de l'exposition à l'aflatoxine $\mathrm{B}_{1}$, il existe un site unique de mutations sur le gène P53 (codon $n^{\circ} 249$, mutation de $G$ en $T$ ) dont l'étude permettra éventuellement de déterminer le mécanisme de ce type de cancers (Hsu et al., 1991).

Dans le même ordre d'idée, nous avons déterminé les types de mutation sur les gènes P53 mutés dans les tumeurs de la peau de malades XP. Nous savons que les ultraviolets induisent essentiellement des mutations $\mathrm{C}$ vers $\mathrm{T}$ dont 20 à $30 \%$ sont des mutations doubles $\mathrm{C}-\mathrm{C}$ vers T-T. Ce type de spectre est vraiment spécifique de la mutagénèse UV (Madzak and Sarasin, 1991). Dans les tumeurs XP, qui sont dues dans $99 \%$ des cas à la présence de lésions UV-induites non réparées, nous avons trouvé qu'environ la moitié des tumeurs contenaient des mutations sur le gène P53. Toutes ces mutations étaient localisées au niveau de sites pyrimidine-pyrimidine qui représentent les points chauds des lésions induites par les UV. La grande majorité de ces mutations étaient de type $\mathrm{C}$ vers $\mathrm{T}$ et environ $70 \%$ étaient des mutations doubles $\mathrm{C}-\mathrm{C}$ vers $\mathrm{T}-\mathrm{T}$ (N. Dumaz et L. Daya-Grosjean, résultats non publiés). Ce résultat confirme clairement le rôle des lésions induites par les UV comme agent mutagène du gène P53 (et aussi des oncogènes $\underline{\text { ras }}$ ) et donc comme agent initiateur des cancers cutanés.

L'étude des oncogènes et des gènes supresseurs de tumeurs, non seulement conduit à une meilleure compréhension du mécanisme moléculaire du développement des cancers chez l'homme, mais pourra éventuellement permettre de déterminer l'agent responsable de l'initiation du processus tumoral. 


\section{Références :}

H.G. SUAREZ, P. NARDEUX, Y. ANDEOL and A. SARASIN Multiple activated oncogenes in human tumors.

Oncogene Res., 1987, 1:201-207.

H.G. SUAREZ, L. DAYA-GROSJEAN, D. SCHLAIFER, P. NARDEUX, G. RENAULT, J.L. BOS and A. SARASIN

Modified oncogenes in human skin tumors from a repair deficient syndrome, xeroderma pigmentosum.

Cancer Res., 1989, 49:1223-1228.

L.J. VAN DER LUBBE, H.J.M. ROSDORFF, J.L. BOS and A.J. VAN DER EB. Activation of $\mathrm{N}$-ras induced by ultraviolet irradiation in vitro. Oncogene Res., 1988, $3: 9-20$.

L. DAYA-GROSJEAN, C. ROBERT, C. DROUGARD, H.G. SUAREZ and A. SARASIN

High mutation frequency in ras genes of skin tumors isolated from DNA-repair deficient xeroderma pigmentosum patients. Cancer Res., 1993, in press.

L. HARTWELL

Defects in a cell cyle checkpoint may be responsible for the genomic instability of cancer cells.

Cell, 1992, 71:543-546.

M.B. KASTAN, Q. ZHAN, W.S. EL-DEIRY, F. CARRIER, T. JACKS, W.V. WALSH, B.S. PLUNKETT, B. VOGELSTEIN and A.J. FORNACE, Jr. A mammalian cell cycle checkpoint pathway utilizing p53 and GADD45 is defective in ataxia-telangiectasia.

Cell, 1992, 71:587-597.

C. CARON DE FROMENTEL and T. SOUSSI.

TP53 tumor suppressor gene : a model for investigating human mutagenesis.

Genes, Chromosomes \& Cancer, 1992, 4:1-15.

I.C. HSU, R.A. METCALF, T. SUN, J.A. WELSH, N.J. WANG and C.C. HARRIS.

Mutational hotspot in the p53 gene in human hepatocellular carcinomas.

Nature, 1991, 350:427-428.

C. MADZAK and A. SARASIN.

Mutation spectrum following transfection of ultraviolet-irradiated single-stranded or double-stranded shuttle vector DNA into monkey cells.

J. Mol. Biol., 1991, 218:667-673. 